\title{
Parallel algorithms for generating combinatorial objects on linear processor arrays with reconfigurable bus systems*
}

\author{
P THANGAVEL \\ Department of Computer Science, University of Madras, Chepauk, \\ Madras 600005, India \\ e-mail: thang@unimad.ernet.in
}

MS received 21 November 1996; revised 18 October 1997

\begin{abstract}
A bus system whose configuration can be dynamically changed is called reconfigurable bus system. In this paper, parallel algorithms for generating combinations, subsets, and binary trees on linear processor array with reconfigurable bus systems (PARBS) are presented.
\end{abstract}

Keywords. Parallel algorithms; recontigurable bus systems; combinations; subsets; binary trees.

\section{Introduction}

Generating combinations and permutations are common combinatorial problems. We denote $C m n$ to be the number of combinations obtained by choosing $m$ objects out of $n$ objects. Sequential algorithms optimally take a time of $O\left(m^{*} \mathrm{Cmn}\right)$. Akl et al $(1989 / 90)$ have presented a parallel algorithm, using an Exclusive Read, Exclusive Write, Parallel Random Access Machine (EREW PRAM) model, which takes $O(\mathrm{Cmn})$ time optimally. Section 3 considers the combinations generation using an $m$-processor linear PARBS. This algorithm outputs a combination in constant time. The combinations are generated in lexicographic ascending order. All the Cmn combinations can be generated and output in a time of $O(\mathrm{Cmn})$. Though our algorithm takes the same time as that of Akl et al (1989/90), we present it because of the implementation feasibility of the PARBS model.

Generation of subsets has applications in subset sum, knapsack, base enumeration and minimal covering problems (Stojmenovic \& Miyakawa 1983) A cost-optimal systolic algorithm for generating subsets was presented in Tsay \& Lee (1994). In $\$ 4$ we present a cost-optimal parallel algorithm using linear PARBS.

The problem of enumerating $n$-noded binary trees have been studied by introducing integer sequences or permutations, which characterize trees in a combinatorial way. One

*Preliminary version of this paper has been presented at the 6th National Seminar on Theoretical Computer Science, held at Bhanasthali Vidyapith, Aug. 8-12, 1996 
such sequence called $P$-sequence was introduced by Pallo \& Racca (1985). They have provided a simple algorithm to generate $P$-sequences lexicographically. In $\S 5$ an efficient and simple parallel algorithm for generating $P$-sequences is proposed which runs on a linear PARBS.

Zerling (1985) reported a new approach for generating rooted ordered binary trees with $n$-nodes in some order by using rotations. He established a $1-1$ correspondence between binary trees and codewords related to rotations, called rotational admissible codewords for binary trees, and presented a recursive algorithm to generate these codewords. Er (1989) later presented faster recursive algorithm to generate these codewords. Recently Makinen (1991) has given a non-recursive algorithm. All the above algorithms take $O(n)$ time in the worst case to produce the next codeword from the previous one. In $\$ 6$ a simple parallel algorithm for generating rotational-admissible codewords based on the non-recursive algorithm of Makinen (1991) is proposed. This algorithm runs on linear PARBS.

In the next section the PARBS model is introduced.

\section{The PARBS model}

A reconfigurable bus system is a bus system whose configuration can be dynamically changed. Processors in PARBS are assumed to operate in single instruction multiple data (SIMD) mode; that is they perform the same instruction synchronously (at times only very few processors may be active but this will be explicitly specified in the instruction). In a single unit of time, each processor can either select a switch configuration, perform a read or write operation on the bus, or perform a constant-time computation on its local data. The model allows several processors to read the same bus component, but does not allow more than one processor to write on the same bus component at the same time. By giving specific instructions, the processors reading from a bus can be restricted. For example, only when a particular condition is satisfied the processor may read the bus. Each processor may have its own built-in ports. These ports can be dynamically connected in pairs based on computational needs. When a bus configuration is established, processors that are attached to the same bus can communicate with one another with the above restrictions. It is assumed that such a communication takes one unit of time. This basic assumption on PARBS models is acceptable because of the gap between signal propagation velocity and reconfiguring rates. With the current hardware technology, signal propagation velocity is very close to its limit (velocity of light), whereas the reconfiguration rates are bounded by both theoretical and technological restrictions. Thus the time for the switch state to change can be orders of magnitude longer than the time for the signal to traverse a distance equal to the diameter of a switch. It is also generally assumed that each processor can perform basic arithmetic and logical operations in unit time (Schuster 1991; Wang 1991).

The linear PARBS model can be described as follows: It consists of $n$-processors, $P_{1}$, $P_{2}, \ldots, P_{N}$. Each processor is connected to its neighbouring processors. The connections are made through two built-in ports denoted by $N$ and $S$ (or $L$ and $R$ ) of each processor. A local switchable network could dynamically change the configuration of the system. It is controlled by the processor itself. Figure 1 shows such a system with $n=5$. 

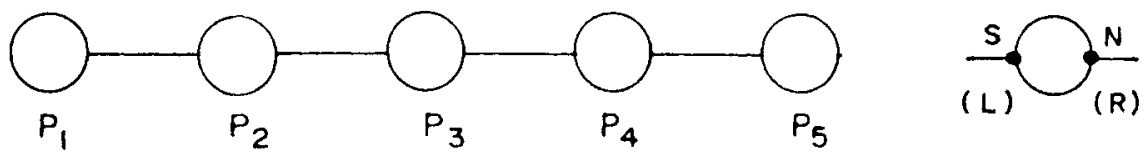

Figure 1. A 5-processor linear PARBS. Here $P_{\imath}$ 's-processors and $\mathbf{S}, \mathbf{N}(\mathrm{L}, \mathbf{R})$ are built-in ports.

\section{Generation of combinations in a linear PARBS}

Consider a linear PARBS with $m$-processors, each processor having six registers $C, L, B$, $R, I$ and $J$. In the registers $C$ and $L$, the components of current (which is used to generate the next one) and last combinations can be stored. Register $B$ is used to store a boolean value by comparing $C$ and $L$. Register $R$ contains a copy of $B$ of next processor. Registers $I$ and $J$ are used to store the indices of processors. Each combination is generated using its previous combination $C_{i}, i=0,1,2, \ldots, m-1$, and last combination $L_{i}\left(L_{i}=n-m+i+1\right)$, $i=0,1,2, \ldots, m-1$, in lexicographic order. Here, assume that we want to generate combinations of $m$ integers from the first $n$ integers $(1<m<n)$. Then it could be easily extended to an array of $n$ elements by considering them as indices. The algorithm consists of the following steps.

Algorithm 1. Parallel generation of combinations.

Step 0: Initially $C_{j}=j+1 ; L_{j}=n-m+j+1 ; I_{j}=j$ and $J_{j}=0$ are stored on corresponding registers of each processor $P_{j}, j=0.1,2, \ldots m-1$.

Step 1: Set the boolean variable $b_{j}$ with 1 , if $C_{j}=L_{j}$, otherwise with 0 (if $C_{j}<L_{j}$ ) and store it in register $\mathrm{B}$ of each processor $P_{j}, 0 \leq j \leq m-1$.

Step 2: If $b_{m-1}=0$, then set $C_{m-1}=C_{m-1}+1$ and store it in register $C$ of processor $P_{m-1}$. Go to step 8 .

Step 3: If $b_{0}=1$ then, go to step 8 (the input combination is the last one i.e., same as $L_{j}$ ). Step 4: Each processor $P_{j}, j=1,2, \ldots, m-1$, sends a copy of $b_{j}$ to processor $P_{j-1}$; say $r_{j-1}$, stored in $R$.

Step 5: Each processor $P_{i}$ for which $b_{i}=1$ connects port $L$ to port $R$, for $1<i<m-1$.

Step 6: Processor $P_{j}$, for which $\bar{b}_{j} \cdot r_{j}=1$, broadcasts $C_{j}$ and $I_{j}$ to processors $P_{k}, k=j+1$, $j+2, \ldots, m-1$, and received in registers $C$ and $J$ respectively.

Step 7: Each processor $P_{k}$ for which $r_{k}=1$ sets $C_{k}=C_{k}+I_{k}-J_{k}+1$, and stored in register $C$.

Step 8: Each processor $P_{k}, k=0,1, \ldots, m-1$ prints its register $C$ value. Then go to step 1 .

To prove the correctness we proceed as follows: We can make the following observations from the lexicographic ascending order of combinations. If $C_{0}, C_{1}, \ldots, C_{m-1}$ and $L_{0}, L_{1}, \ldots, L m-1$ are the current and last combinations then we have (i) $C_{i}<C_{i+1}$, for $i=0,1, \ldots m-2$, and (ii) $C_{i} \preceq L_{i}$, for $i=0,1, \ldots m-1$.

Lemma 1. There exists a unique index $j$ such that $C_{k}=L_{k}$ for $j \leq k \leq m-1$. 
Proof. Let $j$ be the smallest index such that

$$
C_{j}=L_{j} \text { (i.e., } b_{1}=b_{2}=\cdots=b_{j-1}=0 \text { ). }
$$

If $C_{j+1} \neq L_{j+1}$ then $C_{j+1}<L_{j+1}$ (by observation (ii)). From observation (i), $C_{j}<C_{j+1}$ and since $L_{j}<L_{j+1}$, we have $C_{j+1} \preceq L_{j}\left(L_{j}\right.$ and $L_{j+1}$ are consecutive), i.e., $C_{j+1} \preceq C_{j}$, which is a contradiction, therefore

$$
C_{j+1}=L_{j+1} \text {. }
$$

By induction $C_{k}=L_{k}$ for all $k>j$.

If $b_{0}=1$ then $C_{0}=L_{0}$, then $C_{1}=L_{1}, C_{2}=L_{2}, \ldots, C_{m-1}=L_{m-1}$ (by lemma 1 ). This implies that the current combination is the same as the last combination (step 3 ). If $b_{m-1}=0$, there is no $C_{j}=L_{j}$, for $j=0,1, \ldots, m-1$, then the processor $P_{m-1}$ increments its component to produce the next combination in order (step 2). By lemma 1 there exists a unique index $j$ such that the sequence $b_{0}, b_{1}, \ldots, b_{m-1}$ will be of the form $0,0, \ldots, 0,1,1, \ldots, 1$, (first 1 is in processor $P_{j+1}$ ). This ensures that there is a unique processor $P_{j}$ for which $\bar{b}_{j} \cdot r_{j}=1$, therefore this processor can broadcast $C_{j}$ and $I_{j}$ in step 6. It also proves that step 5 can create a bus starting from processor $P_{j}$ until processor $P_{m-1}$. To prove the correctness of step 7, we prove the following lemma.

Lemma 2. Let $C_{0}, C_{1}, \ldots, C_{m-1}$ and $C 1_{0}, C 1_{1}, \ldots, C 1_{m-1}$ be the current input and output combinations of the above algorithm. Then the two combinations are in lexicographic ascending order.

Proof. Consider the boolean sequence

$$
b_{0}, b_{1}, \ldots, b_{m-1}=0,0, \ldots, 0,1, \ldots, 1,
$$

(first 1 is at processor $P_{j+1}$ ). Then from the algorithm we can see that

$$
\begin{aligned}
& C 1_{i}=C_{i} \quad \text { for } i=0,1, \ldots, j-1 \\
& C 1_{j}=C_{j}+1>C_{j}
\end{aligned}
$$

Hence the two combinations are in lexicographic ascending order.

It can be easily seen that each step of the algorithm takes only a constant amount of time as the broadcast operation takes constant time. Thus we can generate the next combination from the previous one in constant time. The algorithm can be made adaptive (i.e., to run on an array with $k>m$ processors), if we divide the linear array into $k / m$ groups of $m$ processors such that each group can produce an interval of consecutive $m$-combinations. Note that in such cases we need to change the input (current and last) combinations correspondingly.

\section{Parallel generation of subsets}

We assume that each processor in the linear array has registers $A, B, C$, and $D$. Register $A$ is used to store the elements of the current subset. $B, C$ and $D$ are used to store boolean 
values. Now we shall present a parallel algorithm for generating all subsets in lexicographic ascending order of the set $\{1,2, \ldots, n\}$.

Algorithm 2. Parallel generation of subsets.

Step 1. Set $a_{1}=1, a_{i}=0$, for $i>1$, and $b_{i}=c_{i}=d_{i}=0$, for all $i$, and store them in registers $A, B, C$ and $D$ respectively.

Step 2. Each processor $P_{i}$ prints $a_{i}$ for which $a_{i} \neq 0$.

Step 3. If $a_{1}=n$ then stop.

Step 4. Set $b_{i}$ with 1 , if $a_{i}=0$, and 0 otherwise, and $c_{i}$ with 1 if $a_{i}=n$, and 0 otherwise.

Step 5. Each processor $P_{i}$ sends a copy of $c_{i}$ to processor $P_{i-1}$, received in $D$, for $i=$ $2,3, \ldots, n$. Processor $P_{i}$ for which $c_{i} \cdot \bar{d}_{i}=1$, sets $a_{i}=a_{i}+1$.

Step 6. Processor $P_{i}$ for which $c_{i}=1$, sets $a_{i}=0$.

Step 7. Each processor $P_{i}$ for which $\bar{d}_{i}=0$, connects port $L$ with $R$. Processor $P_{i}$ for which $d_{i}=1$ sends $d_{i}$ value which is received in register $D$ of $P_{1}$.

Step 8. If $D_{1}=0$, then each processor $P_{i}$ sends a copy of register $B$ value to $P_{i+1}$ for $1 \leq i<n$, received in register $D$. Then processor $P_{i}$ for which $b_{i} \cdot \bar{d}_{i}=1$ sets $a_{i}=a_{i-1}+1$. Then go to step 2 .

Since each subset either contains only one $n$ or none, there could be only one $i$ for which $c_{i}=1$. This ensures that there could be atmost one processor for which $d_{i}=1$ in step 7 . In this case the entry prior to $n$ is incremented by 1 (step 5) and the entry corresponding to $n$ is set to zero (step 6) to get the next subset. Otherwise (if $n$ is not a member of current subset), the processor for which $b_{i} \cdot \bar{d}_{i}=1$ sets its component of the next subset to $a_{i-1}+1$. The algorithm generates subsets of the set $\{1,2,3,4\}$ in the following order:

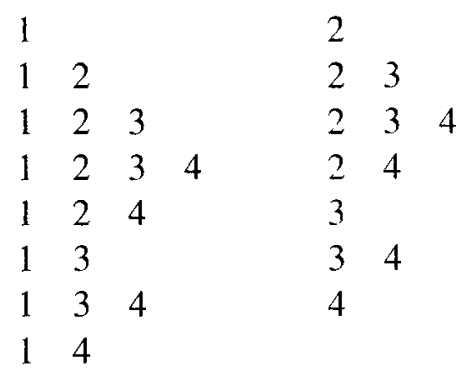

It can be easily seen that the algorithm takes only constant time to generate the next subset from the previous one.

\section{Generating binary trees using $P$-sequences}

First we shall introduce $P$-sequences. Let $r_{T}$ be the degree of the root of the binary tree $T$. Denote by $T_{L}$ and $T_{R}$, the left and right subtrees of $T$. Two orders, namely A-order and B-order proposed in Pallo \& Racca (1985), used for the generation of all shapes of binary trees with $n$ internal nodes, are defined as follows: 


\section{DEFINITION 1}

Two trees $T$ and $T^{\prime}$ are in A-order, denoted by $T<T^{\prime}$ if (1) $|T|<\left|T^{\prime}\right|$ or (2) $|T|=\left|T^{\prime}\right|$ and $T_{L}<T_{L}^{\prime}$ or (3) $|T|=\left|T^{\prime}\right|$ and $T_{L}=T_{L}^{\prime}$ and $T_{R}<T_{R}^{\prime}$ where $|T|$ is the number of leaves in $T$.

\section{DEFINITION 2}

Two trees $T$ and $T^{\prime}$ are in B-order, denoted by $T<T^{\prime}$ if (1) $r_{T}<r_{T^{\prime}}$ or (2) $r_{T}=r_{T^{\prime}}$ and $T_{L}<T_{L}^{\prime}$ or (3) $r_{T}=r_{T^{\prime}}$ and $T_{L}=T_{L}^{\prime}$ and $T_{R}<T_{R}^{\prime}$. $P$-sequences are used to generate binary trees in B-order. Now we define $P$-sequences as follows:

\section{DEFINITION 3}

For a given binary tree $T$, the $P$-sequence of $T$ is the integer sequence $\left(p_{T}(1), p_{T}(2), \ldots\right.$, $\left.p_{T}(|T|-1)\right)$ where $p_{T}(i)$ is the number of internal nodes $O$ written before the leaf $i$ in the Polish notation of $T$.

Theorem 1 (Pallo \& Racca 1985). An integer sequence $\left(p_{1}, p_{2}, \ldots, p_{n}\right)$ is the $P$ sequence of a binary tree with $n$ internal nodes if and only if

(1) for all $i=1,2, \ldots, n, p_{i}<p_{i+1}$,

(2) $p_{n}=n$, and

(3) for all $i=1,2, \ldots, n, p_{i}>i$.

Theorem 2 (Pallo \& Racca 1985). Given two binary trees $T$ and $T^{\prime}$ such that $|T|=\left|T^{\prime}\right|$, $T$ and $T^{\prime}$ are in $B$-order if and only if $p_{T}$ is lexicographically less than $p_{T^{\prime}}$.

The algorithm proposed here is based on the sequential generating algorithm in Pallo \& Racca (1985). It is worth reproducing the same here.

\section{Generating algorithm}

Begin with $\left(p_{1}, p_{2}, \ldots, p_{n}\right)=(1,2, \ldots, n)$

while $i=\max \left(k \mid p_{k}<n\right)$ exists do

$$
p_{i}=p_{i+1}
$$

for $j=i+1$ to $n$ do $p_{j}=\max \left(p_{i}, j\right)$ end do end do

We consider a linear PARBS with $n$ processors. Each processor has registers $B, C, D$ and $I$. $B$ and $C$ are used to store boolean values. $D$ is used to store $P$-sequence elements and $I$ is for index $i$ of the corresponding processor $P_{i}$. Initially assume that $d_{i}=i$ and $I_{i}=i$ for $i=1,2, \ldots, n$. A parallel algorithm for generating $P$-sequences in lexicographic order is given in algorithm 3 .

Algorithm 3. Parallel generation of $P$-sequences.

Step 1. Set $d_{i}=i$ and $I_{i}=i$ and store them in registers $D$ and $I$ of each processor $P_{i}$, $i=1,2, \ldots, n$.

Step 2. Set the boolean variable $b_{i}$ with 1 , if $d_{i}=n, 0$ otherwise. 
Step 3. If $b_{1}=1$ then stop.

Step 4. Each processor $P_{i}$ sends a copy of $b_{i}$ to processor $P_{i-1}$, say $c_{i-1}$, stored in $C$, for $i=2,3, \ldots, n$.

Step 5. Each processor $P_{i}$, for which $b_{i}=1$, connects port $L$ to port $R$.

Step 6. Processor $P_{i}$, for which $\bar{b}_{i} \cdot c_{i}=1$, sets $d_{i}=d_{i+1}$ and broadcasts $d_{i}$ to processors $P_{i+1}, P_{i+2}, \ldots, P_{n}$, stored in register $D$.

Step 7. Each processor $P_{i}$ for which $b_{i=1}$, sets $d_{i}$ with $d_{i}$, if $d_{i}>I_{i}$, and $I_{i}$ otherwise.

Step 8. Each processor $P_{i}$ writes $d_{i}, i=1,2 \ldots, n$ (next $P$-sequence). Then go to step 2.

Note that there is a unique processor $P_{i}$ for which $\bar{b}_{i} \cdot c_{i=1}$, as the sequence $b_{i}, i=$ $1,2, \ldots, n$ will be of the form $0,0, \ldots .0,1,1, \ldots, 1$ which follows from the definition of $P$-sequence. It takes only constant time to produce the next $P$-sequence from the previous one.

\section{Parallel generation of binary trees using rotational admissible codewords}

A rotational-admissible codeword is a sequence of integers obtainable by atation-based coding method. A codeword $\left(x_{0}, x_{1}, \ldots, x_{n-1}\right)$ is rotational admissible if $x_{0}=0$ and for each $i=1.2, \ldots, n-1$, we have $0 \leq x_{i} \leq x_{i-1}+1$. It has been shown that there is a one to one correspondence between codewords and binary trees (Zerling 1985). In this section we shall present a parallel algorithm based on Makinen's (1991) non-recursive algorithm, which is worth reproducing. It uses an array $x$ containing the codewords to be generated, and $x$ is initialised by zeroes. Basically the algorithm adds one to the previous codeword. Variable pos shows the position of $x$ in which we perform the addition, and it is initialized to contain the value $n-1$.

Procedure non-recursive Enumerate

begin

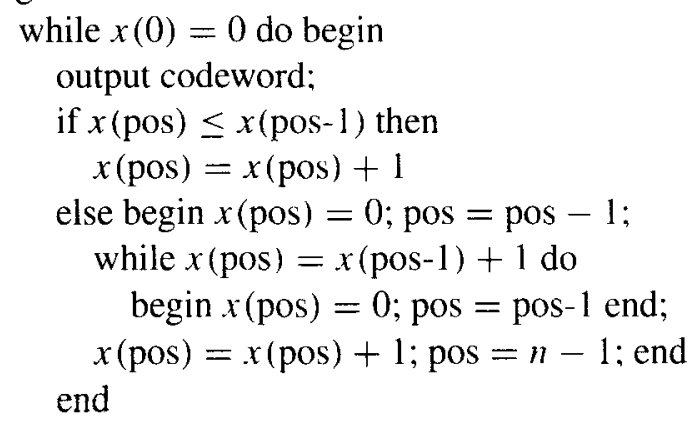

end;

Consider a linear PARBS with each processor having registers $A, B, C$ and $X . X$ is used to store the components of the codeword. Register $A$ is also used to store codewords. $B$ and $C$ are used to store boolean values. The algorithm is given below.

Algorithm 4. Parallel generation of rotational-admissible codewords

Step 1. Each processor $P_{i}$ sets $x_{i}=0$, for $0 \leq i \leq n-1$. 
Step 2. If $x_{0}=0$, then each processor $P_{i}$ writes $x_{i}$, for $0 \leq i \leq n-1$. Otherwise stop. Step 3. Processor $P_{n-2}$ sends a copy of $x_{n-2}$ to processor $P_{n-1}$, received in register $A$. Step 4. If $x_{n-1} \leq a_{n-1}$, processor $P_{n-1}$ sets $x_{n-1}=x_{n-1}+1$, and then go to step 2 .

Step 5. Each processor $P_{i}, 0 \leq i \leq n-2$, sends a copy of $x_{i}$ to processor $P_{i+1}$, received in register $A$.

Step 6. Processor $P_{n-1}$ sets $x_{n-1}=0$.

Step 7. Each processor $P_{i}$ sets $a_{i}=a_{i}+1$, stores in register $A$ itself.

Step 8. Each processor $P_{i}$ sets $b_{i}$ with 1 , if $a_{i}=x_{i}$ otherwise 0 .

Step 9. Each processor $P_{i}$ connects port $L$ to port $R$ for which $b_{i}=1$. Processor $P_{n-1}$ sends a 1 signal through this bus, which is received in register $C$ of each processor in the bus. Disconnect all switches.

Step 10. Each processor $P_{i}$ for which $\bar{b}_{i} \cdot c_{i}=1$ sets $x_{i}=0$ and $\bar{b}_{i} \cdot c_{i}=1$ sets $x_{i}=x_{i}+1$. Then go to step 2.

It is worth noting that there is a unique processor $P_{i}$ for which $\bar{b}_{i} \cdot c_{i}=1$. The above algorithm is repeated until $x_{0}=0$. It can be easily seen that the algorithm takes only constant amount of time to produce the next codeword from the previous one.

\section{Conclusion}

We have presented parallel algorithms for generating combinatorial objects combinations, subsets, and binary trees based on $P$-sequences and rotational admissible codewords. Our algorithms take only constant time to produce next object from the previous one. There are many combinatorial open problems for which we can design fast parallel algorithms by exploiting the flexible nature of PARBS models.

\section{References}

Akl S G, Gries D, Stojmenovic I 1989/90 An optimal parallel algorithm for generating combinations. Inf. Process. Lett. 33: 135-139

Er M C 1989 A new algorithm for generationg binary trees using rotations. Comput.J. 32: 470-473

Makinen E 1991 Efficient generation of rotational-admissible codewords for binary trees. Comput. J. 34: 379

Pallo J, Racca R 1985 A note on generating binary trees in A-order and B-order. Int. J. Comput. Math. 18: 27-39

Schuster A 1991 Dynamic reconfiguring networks for parallel computers: algorithms and complexity bounds. PhD thesis, Hebrew University, Jerusalem

Stojmenovic I, Miyakawa M 1983 Applications of subset generating algorithm to base enumeration, knapsack and minimal covering problems. Comput. J. 1: 65-70

Tsay J C, Lee W P 1994 A cost-optimal systolic algorithm for generating subsets. Int. J. Comput. Math. 50: 1-10

Wang B F 1991 Configurational computation: a new algorithm design strategy on processor arrays with reconfigurable bus systems. PhD thesis, National Taiwan University, Taipei

Zerling D 1985 Generating binary trees using rotations. J. Assoc. Comput. Mach. 32: 694-701 